\title{
The LHCb trigger: algorithms and performance
}

\author{
Hugo Ruiz, on behalf of the LHCb collaboration \\ Universitat de Barcelona, Diagonal 647, 08028 Barcelona, Spain
}

\begin{abstract}
This article describes the hardware architecture of the LHCb trigger system, as well as the algorithms that it executes in order to identify the relevant B meson decays. The expected performance on simulated data and the trigger commissioning status and plans are also presented.

The LHCb trigger consists of two phases: the Level 0 and the High Level Trigger. The former is synchronous and implemented in custom electronics, and it reduces the rate to $1 \mathrm{MHz}$. The High Level Trigger is a C++ algorithm running in a farm of about 1000 16-core computing nodes and further reducing the rate to $2 \mathrm{kHz}$.

The Level 0 efficiency computed on B meson events that would be offline-selected range between $90 \%$ for decays containing muons and $50 \%$ for purely hadronic decays, the later suffering from higher light quark backgrounds. Overall High Level Trigger efficiencies range between 60 and $80 \%$.
\end{abstract}

Key words:

Trigger system, Trigger algorithms, B meson physics, LHCb experiment, Data acquisition system

\section{Introduction}

The LHCb detector is ready to exploit the B-physics potential of the LHC. At the luminosity chosen for the $\mathrm{LHCb}$ interaction point of $2 \cdot 10^{32} \mathrm{~cm}^{-2} \mathrm{~s}^{-1}$, the rate of events with charged particles within the detector acceptance is expected to be $10 \mathrm{MHz}$ [1]. One of the challenging requirements of $\mathrm{LHCb}$ is a trigger system capable to reduce the rate down to the $2 \mathrm{kHz}$ allowed by the long-term data storage resources [2], while providing a good efficiency on interesting $\mathrm{B}$ decays.

As the rate of $\mathrm{b}$ quark production will be as high as 100 $\mathrm{kHz}$, the LHCb trigger aims for a subset of the B decay modes. Two main signatures allow identifying particles from B decays: relatively high transverse momentum $\left(p_{T}\right)$ and non-null impact parameter (IP) with respect to the proton-proton interaction vertex.

\section{Trigger architecture}

The LHCb trigger is composed of two levels, called Level 0 (L0) and High Level Trigger (HLT). L0 is synchronous and implemented in custom electronics, and reduces the rate to $1 \mathrm{MHz}$. The HLT is a $\mathrm{C}++$ algorithm running in about 1000 16-core computing nodes forming the Event Filter Farm (EFF).

Email address: hugo.ruiz@cern.ch (Hugo Ruiz, on behalf of the $\mathrm{LHCb}$ collaboration)
L0 uses information from selected sub-detectors, which are read-out at $40 \mathrm{MHz}$ : the calorimeters and muon chambers to provide high $p_{T}$ candidates, and two dedicated layers of the vertex detector to provide a fast estimation of the number of proton-proton interactions that occurred in the bunch crossing.

L0 positive decisions are sent back to the front-end electronics of all the sub-detectors, which pick-up the pieces of the relevant events from buffers and send them through a read-out network to the EFF. The network is built on commercial gigabit Ethernet components and allows a maximum throughput of $50 \mathrm{~GB} / \mathrm{s}$.

The HLT has hence access to information from the whole detector. However, a complete event reconstruction is not feasible at $1 \mathrm{MHz}$. The HLT reconstructs first regions of interest defined by the L0 candidates. Events where candidates do not satisfy the required conditions when tracking and vertexing information is added are promptly discarded.

\section{L0 trigger}

The L0 trigger decision is taken by the L0 Decision Unit, based on the following pieces of information provided by the trigger boards:

- Calorimeter clusters classified as electrons $\left(e^{ \pm}\right)$, photons $(\gamma)$, neutral pions $\left(\pi^{0}\right)$ or charged hadrons $\left(h^{ \pm}\right)$ according to the energy deposition in the different calorimeter layers. 
- Muon $\left(\mu^{ \pm}\right)$and dimuon $\left(\mu^{+} \mu^{-}\right)$candidates.

- The result of a fast proton-proton interaction vertex search based on two dedicated layers of the vertex detector (the Pileup System).

- The multiplicities in the Pileup System and in a scintillator layer in front of the calorimeter (SPD).

In the calorimeter, $2 \times 2$-cell clusters are formed. For the particular case of hadronic clusters, the transverse energy $\left(E_{T}\right)$ deposited in the ECAL cells in front of the relevant HCAL cells is also added to compute the $E_{T}$ of the candidate. The relative precision on $E_{T}$ of the hadronic clusters is roughly $\delta E_{T} / E_{T} \sim 30 \%$.

For building muon candidates, straight segments are searched for in the four muon stations downstream of the calorimeter, where the occupancy is relatively low. The search is performed within projective towers, under the assumption that the muon tracks roughly point to the interaction point. The segments found are confirmed by looking for a compatible hit in the muon chamber upstream of the calorimeter. The momentum is then estimated by using a look-up table built under the assumption that the muon tracks originate at the interaction point. The resolution obtained is $\delta p / p \sim 20 \%$.

A positive decision is taken when a calorimeter or muon candidate above the $p_{T}$ or $E_{T}$ threshold is found. The threshold values for different types of candidates are shown in Table 1.

Table 1: $p_{T}$ cuts and rates of the different L0 trigger lines.

\begin{tabular}{|c|c|c|c|c|c|c|}
\hline L0 line & $h^{ \pm}$ & $\mu^{ \pm}$ & $\mu^{+} \mu^{-}$ & $e^{ \pm}$ & $\gamma$ & $\pi^{0}$ \\
\hline$p_{T}>(\mathrm{GeV})$ & 3.5 & 1.3 & $\Sigma>1.5$ & 2.6 & 2.3 & 4.5 \\
\hline Rate $(\mathrm{Hz})$ & 700 & \multicolumn{3}{|c|}{200} & \multicolumn{3}{|c|}{300} \\
\hline
\end{tabular}

If the running conditions require it, vetoes on the number of interaction vertices and on multiplicities can be applied to any L0 line. This would allow removing events which are harder to process at the HLT.

\section{The HLT trigger}

The HLT algorithm [4] is divided in two sequential phases called HLT1 and HLT2. HLT1 applies a progressive, partial reconstruction seeded by the L0 candidates. Different reconstruction sequences (called alleys) with different algorithms and selection cuts are applied according to the L0 candidate type.

In general, track segments pointing to candidates are first searched for at the main tracking stations. If they are found, the corresponding L0 $p_{T}$ cut is applied again, now with $\delta p / p \sim 3 \%$.

Finally, matching segments are searched for at the vertex detector (VELO). In order to minimize the time consumption, a fast reconstruction is performed first by using the half of the VELO sensors that measure the $R$ coordinate (distance to the beam axis) [3]. This allows reconstructing the so-called VELO two-dimensional (2D) tracks. If any of such tracks is matched to a L0 candidate, primary vertices are then reconstructed using VELO 2D tracks. For the tracks matched to L0 candidates, the complete 3D reconstruction at the VELO is performed by adding information from the other half of the VELO sensors, which measure the $\phi$ coordinate (the azimuthal angle). If the 3D track is found, then an IP cut is applied (except in the case of some muon lines).

In some alleys, extra tracks forming a good vertex with the original candidate are required before accepting the event. The search of such tracks uses VELO 2D track segments as seeds.

HLT1 selects $20 \mathrm{kHz}$ of events, on which the HLT2 algorithm is then executed. The later consists in its turn of two steps: a whole event reconstruction, very similar to the one to be applied offline, and the so-called HLT2 selections. The latest aim for the following signatures:

- Displaced vertices formed by two, three or four tracks.

- Single muons with high $p_{T}$ and IP

- Pairs of leptons (muons or electrons) with moderate $p_{T}$ and IP and forming a good vertex.

- Leptons (electrons or muons) forming a secondary vertex with an additional track.

- A photon cluster in the calorimeter and a pair of tracks forming a secondary vertex, aiming for radiative B decays $(B \rightarrow X \gamma)$.

- Inclusive $B \rightarrow D X$ and $\Phi \rightarrow K^{+} K^{-}$.

The number of selections, as well as their degree of sophistication will increase as the online tracking is tuned to provide a performance similar to that obtained offline.

\section{Commissioning and start-up strategy}

\subsection{L0 commissioning}

Cosmic ray triggers have been extensively used for alignment and synchronization of LHCb subdetectors since March 2008. The trigger used is the coincidence of signals in the ECAL and the HCAL, obtained by a special configuration of the L0 decision unit.

Cosmic events have allowed synchronizing the L0 trigger path and the L0 electronics for the $e^{ \pm}, \gamma, \pi^{0}$ and $h^{ \pm}$ lines. The algorithms have been checked by comparing the recorded L0 banks with an offline emulation executed on the detector banks collected through the normal data acquisition (DAQ) path.

Cosmic events are not useful to check the L0 muon reconstruction procedure, which assumes that the candidate tracks roughly point to the interaction point. This procedure has been tested with the first particles provided 
by LHC. An example are the beam dumps into a collimator $350 \mathrm{~m}$ upstream of $\mathrm{LHCb}$. About 250 events of this type have been recorded, with about 70 muon tracks reconstructed in each of them.

\subsection{HLT commissioning}

The performance of the HLT algorithms in terms of efficiency, rate reduction and CPU time consumption has been extensively checked on MC simulation.

The HLT services are being commissioned on the EFF by means of the $\mathrm{LHCb}$ full experiment system test. This consists on the injection of Monte Carlo events into the DAQ network for their processing in the EFF. The full system test is being used to deploy the infrastructure for monitoring, configuring and controlling the algorithms, including the propagation of the alignment constants for the reconstruction. In February 2009, the test setup has been successfully used with $2 \mathrm{kHz}$ output rate. It will be systematically used for the commissioning until collisions are provided by the LHC.

$35 \%$ of the CPUs for of the EFF will be deployed by the end of 2009. The rest will be acquired only when the rate of LHC collisions requires it, in order to obtain the most performing equipment within the available budget.

\subsection{Start-up plans}

The LHCb trigger will be configured to collect the nominal $2 \mathrm{kHz}$ since LHC start-up. The priority will be set first to collect trigger-unbiased events for MC tuning. Then, a soft "minimum bias" trigger will be applied by requesting some energy deposition in the calorimeter. This should allow collecting $10^{8}$ events in a week even with a single bunch circulating in each direction. The collected sample will allow performing flavour production studies. Later, priority will be given to muon triggers, which are the most efficient for the channels from which first physics results are expected at $\mathrm{LHCb}$ [5]. The rest of lines will be commissioned and included in the selection progressively.

\section{Performance}

Table 2 shows the L0, HLT and overall efficiencies as expected from Monte Carlo simulation for three important channels for the physics prospects of LHCb. Efficiencies refer to events that pass the offline selection optimized for the relevant physics analyses. A complete detector description, as well as spill-over and pile-up effects are included in the simulation.

The three channels appearing in Table 2 represent three families of decays: with only hadrons; with some leptons; or with a photon in the final state, respectively. L0 and HLT efficiencies are found to be similar for all the decays within a family.

When an event is triggered on a $\mathrm{B}$ meson decay, the other B decay in the event has about $40 \%$ probability of
Table 2: L0, HLT and total trigger efficiencies for three example B decays, with the trigger settings foreseen for nominal luminosity.

\begin{tabular}{|c|c|c|c|}
\hline Decay mode & $\epsilon_{L 0}$ & $\epsilon_{H L T}$ & $\epsilon_{\text {Total }}$ \\
\hline$B_{s} \rightarrow D_{s} \pi$ & $50 \%$ & $80 \%$ & $40 \%$ \\
\hline$B_{s} \rightarrow J / \Psi\left(\rightarrow \mu^{+} \mu^{-}\right) \Phi$ & $90 \%$ & $80 \%$ & $70 \%$ \\
\hline$B \rightarrow K * \gamma$ & $70 \%$ & $60 \%$ & $40 \%$ \\
\hline
\end{tabular}

laying within the $\mathrm{LHCb}$ geometrical acceptance. As a consequence, the $\mathrm{LHCb}$ trigger will provide the unprecedented amount of $\sim 1.5 \cdot 10^{9}$ fully contained, trigger-unbiased $\mathrm{B}$ meson decays per $2 \mathrm{fb}^{-1}$, which is the expected integrated luminosity per year at nominal conditions.

\section{Acknowledgments}

I would like to thank Johannes Albrecht, Xavier Cid, Hans Dijkstra, Jose Angel Hernando, Jaap Panman, Antonio Perez-Calero and Ricardo Vazquez for their help in the preparation of these proceedings and the corresponding oral presentation.

\section{References}

[1] The LHCb collaboration, LHCb Trigger System Technical Design Report, CERN-LHCC-2003-31 (2003).

[2] The LHCb collaboration, LHCb Computing Technical Design Report, CERN-LHCC-2005-19 (2005).

[3] The LHCb collaboration, VELO Technical Design Report, CERN-LHCC-2001-011 (2001).

[4] Johannes Albrecht, The LHCb Trigger, Proceedings of the 8th International Conference on Hyperons, to be published in $\mathrm{Nu}-$ clear Physics B Proceedings Supplements.

[5] Alessio Satti on behalf of the LHCb collaboration, Proceedings of the 19th Hadron Collider Physics Symposium 2008, CERNLHCb-2008-043. 